\title{
The Koala (Phascolarctos cinereus) faecal microbiome differs with diet in a wild population
}

\author{
Kylie L. Brice ${ }^{\text {Corresp., }}$ 1, 2 ， Pankaj Trivedi ${ }^{1,2,3}$ ，Thomas C. Jeffries ${ }^{1,3}$ ， Michaela D. J. Blyton ${ }^{1}$, Christopher Mitchell \\ 1,4 , Brajesh K. Singh ${ }^{1,3}$, Ben D. Moore ${ }^{\text {Corresp. } 1}$ \\ ${ }^{1}$ Hawkesbury Institute for the Environment, Western Sydney University, Penrith, NSW, Australia \\ 2 Bioagricultural Sciences \& Pest Management, Colorado State University, Fort Collins, Colorado, United States \\ 3 Global Centre for Land Based Innovation, Western Sydney University, Penrith, NSW, Australia \\ 4 Environment and Sustainability Institute, University of Exeter, Penryn, Cornwall, United Kingdom \\ Corresponding Authors: Kylie L. Brice, Ben D. Moore \\ Email address: kylie.brice@colostate.edu,b.moore@westernsydney.edu.au
}

Background. The diet of the koala (Phascolarctos cinereus) is comprised almost exclusively of foliage from the genus Eucalyptus (family Myrtaceae). Eucalyptus produces a wide variety of potentially toxic plant secondary metabolites which have evolved as chemical defences against herbivory. The koala is classified as an obligate dietary specialist, and although dietary specialisation is rare in mammalian herbivores, it has been found elsewhere to promote a highly-conserved but low-diversity gut microbiome. The gut microbes of dietary specialists have been found sometimes to enhance tolerance of dietary PSMs, facilitating competition-free access to food. Although the koala and its gut microbes have evolved together to utilise a low nutrient, potentially toxic diet, their gut microbiome has not previously been assessed in conjunction with diet quality. Thus, linking the two may provide new insights in to the ability of the koala to extract nutrients and detoxify their potentially toxic diet.

Method. The 16S rRNA gene was used to characterise the composition and diversity of faecal bacterial communities from a wild koala population $(n=32)$ comprising individuals that predominately eat either one of two different food species, one the strongly preferred and relatively nutritious species Eucalyptus viminalis, the other comprising the less preferred and less digestible species Eucalyptus obliqua.

Results. Alpha diversity indices indicated consistently and significantly lower diversity and richness in koalas eating E. viminalis. Assessment of beta diversity using both weighted and unweighted UniFrac matrices indicated that diet was a strong driver of both microbial community structure, and of microbial presence / absence during across the combined koala population and when assessed independently. Further, Principal Coordinates Analysis based on both the weighted and unweighted UniFrac matrices for the combined and separated populations, also revealed a separation linked to diet. During our analysis of the OTU tables we also detected a strong association between microbial community composition and host diet. We found that the phyla Bacteroidetes and Firmicutes were co-dominant in all faecal microbiomes, with Cyanobacteria also co-dominant in some individuals; however, the E. viminalis diet produced communities dominated by the genera Parabacteroides and/or Bacteroides, whereas the $E$. obliqua-associated diets were dominated by unidentified genera from the family Ruminococcaceae.

Discussion. We show that diet differences, even those caused by differential consumption of the foliage of two species from the same plant genus, can profoundly affect the gut microbiome of a specialist folivorous mammal, even amongst individuals in the same population. We identify key microbiota associated with each diet type and predict functions within the microbial community based on 80 previously identified Parabacteroides and Ruminococcaceae genomes. 


\section{Title page}

2 Title of work reported: The Koala (Phascolarctos cinereus) faecal microbiome differs with diet 3 in a wild population.

4 Authors: Kylie L. Brice ${ }^{1,3^{*}}$, Pankaj Trivedi ${ }^{1,2,3}$, Thomas C. Jeffries ${ }^{1,2}$, Michaela D. J. Blyton ${ }^{1}$, 5 Christopher Mitchell ${ }^{1,4}$, Brajesh K. Singh ${ }^{1,2}$, Ben D. Moore ${ }^{1 *}$.

6 Author affiliations whilst the study was conducted: ${ }^{1}$ Hawkesbury Institute for the Environment,

7 Western Sydney University, Locked bag 1797 Penrith, NSW, 2751, Australia.

$8 \quad{ }^{2}$ Global Centre for Land Based Innovation, Western Sydney University, Locked bag 1797

9 Penrith, NSW, 2751, Australia. ${ }^{3}$ Colorado State University, Bioagricultural Sciences \& Pest Management, C023 Plant Sciences Building, Fort Collins, 80523, Colorado, United States of America. ${ }^{4}$ Environment \& Sustainability Institute, University of Exeter, Penryn, Cornwall, 12 United Kingdom, TR10 9FE.

Corresponding authors: Kylie Brice, ${ }^{3}$ Colorado State University, Bioagricultural Sciences \& Pest Management, C023 Plant Sciences Building, Fort Collins, 80523, Colorado, United States of America. Email: kylie.brice@colostate.edu or kyliebrice72@gmail.com. Phone: +1 9704023556. Ben Moore, ${ }^{1}$ Hawkesbury Institute for the Environment, Western Sydney University, Locked bag 1797 Penrith, NSW, 2751, Australia. Email: b.moore@westernsydney.edu.au, Phone: +61 24570 1384.

Running title: Wild koala microbiomes.

Originality-Significance Statement: This study investigated the gut microbiome of a large cohort of wild (in-situ) koalas ( $\mathrm{n}=32$ ), a vulnerable species that is also a dietary specialist. Significance: We analysed and report diet data, which links diet to individual koala gut microbiomes (reported for the first time). Previous koala gut microbiome studies incorporated three koalas (two wild, one sick and one hit by vehicle, and one captive koala) and did not link the gut microbiome to diet eaten. 
29 Abstract

30 Background.

31 The diet of the koala (Phascolarctos cinereus) is comprised almost exclusively of foliage from the 32 genus Eucalyptus (family Myrtaceae). Eucalyptus produces a wide variety of potentially toxic 33 plant secondary metabolites which have evolved as chemical defences against herbivory. The 34 koala is classified as an obligate dietary specialist, and although dietary specialisation is rare in mammalian herbivores, it has been found elsewhere to promote a highly-conserved but lowdiversity gut microbiome. The gut microbes of dietary specialists have been found sometimes to enhance tolerance of dietary PSMs, facilitating competition-free access to food. Although the koala and its gut microbes have evolved together to utilise a low nutrient, potentially toxic diet, their gut microbiome has not previously been assessed in conjunction with diet quality. Thus, linking the two may provide new insights in to the ability of the koala to extract nutrients and detoxify their potentially toxic diet.

42

\section{Method.}

The 16S rRNA gene was used to characterise the composition and diversity of faecal bacterial communities from a wild koala population $(n=32)$ comprising individuals that predominately eat either one of two different food species, one the strongly preferred and relatively nutritious species Eucalyptus viminalis, the other comprising the less preferred and less digestible species Eucalyptus obliqua.

\section{Results.}

Alpha diversity indices indicated consistently and significantly lower diversity and richness in koalas eating E. viminalis. Assessment of beta diversity using both weighted and unweighted UniFrac matrices indicated that diet was a strong driver of both microbial community structure, and of microbial presence / absence during across the combined koala population and when assessed independently. Further, Principal Coordinates Analysis based on both the weighted and unweighted UniFrac matrices for the combined and separated populations, also revealed a separation linked to diet. During our analysis of the OTU tables we also detected a strong association between microbial community composition and host diet. We found that the phyla Bacteroidetes and Firmicutes were co-dominant in all faecal microbiomes, with Cyanobacteria 
58 also co-dominant in some individuals; however, the E. viminalis diet produced communities

59 dominated by the genera Parabacteroides and/or Bacteroides, whereas the E. obliqua-associated

60 diets were dominated by unidentified genera from the family Ruminococcaceae.

\section{Discussion.}

We show that diet differences, even those caused by differential consumption of the foliage of two species from the same plant genus, can profoundly affect the gut microbiome of a specialist folivorous mammal, even amongst individuals in the same population. We identify key microbiota associated with each diet type and predict functions within the microbial community based on 80 previously identified Parabacteroides and Ruminococcaceae genomes.

\section{Introduction}

Our understanding of the contribution of the gut microbiome to digestive efficiency in vertebrate herbivores, particularly via fermentation and detoxification of low-quality diets, is rapidly improving alongside improved technology for molecular sequencing (Flint and Bayer, 2008b; Kohl and Dearing, 2012; Miller et al., 2014, Suzuki, 2017). Previous investigations into the microbiome have shown that gut microbes are essential to hosts and have identified key species that are thought to have co-evolved with their hosts and which normally maintain host homeostasis but can negatively impact the health and wellbeing of the host when disrupted through illness, antibiotic treatment or dietary dysbiosis (Clarke et al., 2012). To identify the role of gut microbes in host health and wellbeing, many studies create dysbiosis on a large scale through administration of high doses of antibiotics (Kohl et al., 2014d), substantial diet change e.g. from carnivorous to herbivorous (David et al., 2014), or through the addition or removal of toxic components of the diet (Kohl et al., 2014d). Currently, research into the gut microbiomes of specialist folivores is neglected (Barker et al., 2013; Alfano et al., 2015; Barelli et al., 2015).

True obligate dietary specialisation is rare in mammalian herbivores (Shipley et al., 2009) and has been found to promote a highly-conserved but low-diversity gut microbiome in sloths (DillMcFarland et al., 2015). Dietary specialists often rely on plant species that produce potentially toxic plant secondary metabolites (PSMs) which have often evolved as chemical defences against herbivory and reduce consumption by deterrence or reduction of net nutritional benefit of food (Stupans et al., 2001; Moore and Foley, 2005b; Provenza, 2006). However, gut microbes of specialists can sometimes also enhance tolerance of dietary PSMs, facilitating competition-free 
88

89

90

access to food sources (Kohl et al., 2014d). For example, Kohl et al. (2014d) demonstrated how microbes facilitate the intake of toxic PSMs using two populations of the specialist desert wood rat (Neotoma lepida), one experienced and one naïve to feeding on creosote bush (Larrea tridentata), which produces a resin high in the toxic PSM nordihydroguaiaretic acid. Faecal transplantation of microbes from experienced to naïve wood rats increased the tolerance of the latter group to creosote PSMs and antibiotic disruption produced a naïve gut response in experienced wood rats.

The diet of the koala (Phascolarctos cinereus) is comprised almost exclusively of foliage from the eucalypt genus Eucalyptus (family Myrtaceae). However, eucalypts produce a variety of PSMs including terpenes, cyanogenic glucosides, phenolics including condensed and hydrolysable tannins, formylated phloroglucinol compounds (FPCs) and unsubstituted B-ring flavanones (UBFs) which variously act as toxins, feeding deterrents and digestibility-reducers (Moore et al., 2004a; Moore and Foley, 2005b; Marsh et al., 2015; Marsh et al., 2017). Some PSMs, particularly monoterpenes, are potentially bactericidal (Knezevic et al., 2016; Sun et al., 2017), although the koala is a hindgut fermenter and in other hindgut-fermenting marsupials, terpenes are absorbed in the stomach and small intestine, minimising exposure of the gastrointestinal microbiome to these compounds (Foley et al., 1987). Concentrations and types of PSMs vary widely between eucalypt species, between individual trees and from region to region (Moore et al., 2004b; Moore et al., 2004c). Koalas living in regions with different eucalypt communities may therefore face differing nutritional and toxicological challenges (DeGabriel et al., 2009b). Koala microbiomes have not previously been assessed in conjunction with diet quality, although linking the two may provide new insights. The $16 \mathrm{~S}$ rRNA gene has previously been used to describe the microbial community structure of the wild (Barker et al., 2013) and captive (Alfano et al., 2015; Shiffman et al., 2017) koala gut microbiome (Barker et al., 2013; Alfano et al., 2015; Shiffman et al., 2017), finding it to be dominated by bacteria from the phyla Bacteroidetes and Firmicutes.

\section{Background - Cape Otway}

In southern Australia, some koala populations, usually associated with Eucalyptus viminalis (subgenus Symphyomyrtus), have repeatedly increased beyond the carrying capacity of their habitat, resulting in eventual population collapse and mass tree dieback (Martin, 1985a; Whisson et al., 2016). At Cape Otway, Victoria (3850’06” S, 143³0’25” E), 75 koalas were reintroduced 
118 in the 1981 and the population rapidly increased, peaking at densities of up to 18 ha $^{-1}$ in 2013 , 119 followed by a rapid population decline, or crash (Whisson et al., 2016). High juvenile recruitment 120 and low mortality of koalas contribute to this phenomenon, but the high nutritional quality of $E$. 121 viminalis, a highly-preferred food tree which dominates areas experiencing overbrowsing, is a key 122 factor. Eucalyptus viminalis possesses high foliar nitrogen $(\mathrm{N})$ concentrations for a eucalypt and 123 its tannins have a negligible impact on the nutritional availability of that N (DeGabriel et al., 2008; 124 Marsh et al., 2014), although it also possesses high FPC concentrations that can limit koala feeding 125 (Moore et al., 2005). At Cape Otway, a minority of koalas use the generally non-preferred 126 Eucalyptus obliqua (subgenus Eucalyptus), usually in forest patches dominated by that species. 127 Koalas at Cape Otway maintain very small home ranges (0.4-1.2 ha-1; Whisson et al., 2016) so 128 that small scale patchiness in tree species distributions can produce very different home range 129 composition. Trees from the subgenus Eucalyptus ("monocalypts") do not produce FPCs (Eschler 130 et al., 2000), but in contrast to subgenus Symphyomyrtus ("symphyomyrts”), do possess UBFs, 131 which possibly act to deter koalas as they do another eucalypt folivore, the common brushtail possum (Trichosurus vulpecula, Tucker et al., 2010; Marsh et al., 2015). On average, monocalypts also possesses lower foliar available N (AvailN) concentrations, compared with symphyomyrt foliage, due to greater protein precipitation by tannins (Wallis et al., 2010). These patterns present an opportunity to compare and contrast the gut microbial communities of koalas consuming two different eucalypt diets.

Here, we aim to: a) determine the impact of two eucalypt (E. viminalis and E. obliqua) diets on the abundance of dominant bacterial groups within the koala gut and; b) assess the diet quality of these two species. We hypothesised that the gut microbiome of koalas eating different eucalypt diets would differ in community composition. To test our hypothesis, we collected faecal material (pellets) from a single koala population that included individuals believed to consume two different eucalypt species, during the population peak in $2013(\mathrm{n}=14)$, and again, from different individuals, post population collapse in $2015(\mathrm{n}=18)$. We characterised the faecal microbial community as a proxy for gut microbial community composition (Amato et al., 2013; David et al., 2014) using 145 both terminal restriction fragment length polymorphism, and 16S rRNA amplicon sequencing. Diet composition was estimated by faecal plant wax analysis. 


\section{Experimental methods}

149 Detailed experimental procedures can be found in the online version of this article under 150 supplementary information.

\section{Study species}

152 The koala is a specialist folivorous marsupial that is digestively and morphologically adapted to 153 its challenging diet of eucalypt foliage. Koalas have an extended caecum and proximal colon which 154 function as the major site of microbial fermentation (Cork and Warner, 1983a; Snipes et al., 1993). 155 In addition, they selectively retain solutes and small particulate matter in their hindgut for extended 156 periods of up to 213 hours (Krockenberger and Hume, 2007). These adaptations increase the 157 exposure of digesta to microbial fermentation, while reducing the amount of microbial protein lost 158 in faecal matter (Foley and Cork, 1992; Hume, 1993; Hume, 2005). Koala faecal material is 159 expelled as hard, dry pellets.

160

161

162

163

164

165

166

167

168

169

170

171

172

173

174

175

176

\section{Faecal sample collection}

Faecal pellets were collected from single a contiguous koala population occupying a four-square kilometre area of the Cape Otway (38 $50^{\prime} 06^{\prime}$ S S, 14330’25” E) peninsula of Victoria, Australia in two collection years, the first from koalas $(n=14)$ during February and September 2013 (see supplementary information Fig. S1), and the second from koalas ( $\mathrm{n}=18)$ during January 2015( see supplementary information Fig. S2). The peak of the koala population boom occurred at Cape Otway in 2013 and resulted in the eventual death by starvation of hundreds or thousands of koalas (Whisson et al., 2016). In 2013, most koalas in E. viminalis patches had limited or no access to adult foliage and subsisted on epicormic regrowth leaves. These individuals may have experienced some degree of malnourishment. However, by 2015, koala population density had declined, and samples were collected from areas with healthier E. viminalis canopies containing a mixture of adult and epicormic foliage.

Faecal samples were collected from koalas $(n=32)$ inhabiting forest patches locally dominated by or exclusively containing either E. viminalis $(\mathrm{n}=19)$ or E. obliqua $(\mathrm{n}=13$; see supplementary information Fig. S1 \& S2), and in most cases koalas were observed feeding in the trees they were occupying. Koalas in E. viminalis forest at Cape Otway occupy very small ( 0.4 to $1.2 \mathrm{ha}^{-1}$, Whisson et al., 2016) home ranges, allowing us to be confident that E. viminalis was the dominant or only 
177 food source available for those areas, and similarly in E. obliqua areas, E. viminalis was absent or 178 entirely defoliated. In 2013, koalas were present in high densities and signs of overbrowsing or

179

180

181

182

183

184

185

186

187

188

189

190

191

192

193

194

195

196

197

198

199

200

201

202

203

204

205

206

severe defoliation were universal in E. viminalis patches while koalas were much less abundant and overbrowsing was less apparent in E. obliqua patches.

Mats were placed under koalas in trees and checked at intervals of no more than four hours throughout the day. Fresh faecal pellets were counted and collected from mats, placed into ziplock bags and placed on ice until they could be transferred, within 2 hours, into a $-20^{\circ} \mathrm{C}$ freezer for storage. Studies into the impact of storage conditions on results of $16 \mathrm{~S}$ ribosomal RNA (rRNA) sequencing from faecal material have concluded that phylogenetic structure and community diversity are not significantly impacted by either short-term storage at $4{ }^{\circ} \mathrm{C}$ or $20{ }^{\circ} \mathrm{C}(<24 \mathrm{~h})$, or long-term storage at $-20{ }^{\circ} \mathrm{C}$ or $-80{ }^{\circ} \mathrm{C}$ (Carroll et al., 2012, Lauber et al 2010).

Due to the non-invasive nature of faecal collection, faeces have been extensively used to study gut microbial composition and ecology in humans and animals during both health and disease (Turnbaugh et al., 2009a; David et al., 2014; Dill-McFarland et al., 2015; Lichtman et al., 2015). Previous studies have found differences in microbial community composition between gastrointestinal and faecal samples (Stearns et al., 2011; Alfano et al., 2015). The use of faeces as a proxy for the gut microbial community has nonetheless revealed important and biologically meaningful findings in both humans and animals (Amato et al., 2013; David et al., 2014) and is the only non-invasive method available for sample collection from wild animals.

As the field work described in this manuscript did not require either the capture or disturbance of koalas, no animal ethics application or scientific research permit was required. All fieldwork was conducted on private land, meaning that no research permit was required under Victorian National Parks legislation.

\section{Analysis of bacterial community through rRNA gene sequencing and analysis}

Amplification and sequencing of the V4 region of the bacterial 16S rRNA gene was undertaken using a previously established protocol and primers 515F and 806R from Caporaso et al., (2011a). Paired-end 16S rRNA community sequencing was performed using the Illumina MiSeq ${ }^{\circledR}$ platform at the Ramaciotti Centre for Genomics (UNSW, Australia; 2013 koala faecal samples) and the Next-Generation Sequencing Facility at Western Sydney University (Richmond, Australia; 2015 koala faecal samples), using the same protocol, primers and Illumina platform. Analyses of 
207 sequence data were performed using the Quantitative Insights into Microbial Ecology (QIIME)

208 pipeline, version 1.8 (Caporaso et al., 2010b). Sequences were quality-checked and low quality $(<$ 209 Q30) sequences were removed from further analysis. Sequences were aligned against the

210 Greengenes 13_8-release database (DeSantis et al., 2006) and potentially chimeric sequences 211 ( $4 \%$ of total sequences) were removed using Chimera Slayer (Haas et al., 2011). Sequences were 212 aligned (PyNAST, Caporaso et al., 2010a), and clustered (uclust, Edgar, 2010) into operational 213 taxonomic units (OTUs) defined as sharing 97\% sequence identity (hereafter, 'taxa'). Samples 214 were rarefied to the smallest dataset consisting of 107813 sequences, and alpha diversity measures 215 including Chaol (measure of species richness), and Shannon indexes (diversity) were calculated 216 (Good, 1953).

217 Beta diversity and relative abundances of taxa were assessed using the phylogenetic distance-based 218 measurement weighted and unweighted UniFrac (diversity, Lozupone et al., 2011). Taxonomic 219 identities at all levels were assigned by default in QIIME using the Ribosomal Database Project 220 (RDP, Wang et al., 2007). PRIMER v 7.0.13 (Clarke, 1993) and PERMANOVA+ (Anderson, 221 2001) were used to conduct multivariate statistical analysis of the summarised OTU tables 222

\section{Cyanobacteria identity and chloroplast contamination assessment}

To confirm identity of sequences identified as Cyanobacteria, we used the CLC genomics workbench software v 7.5 (CLC bio) to perform a basic local alignment search-nucleotide

234 (BLASTn, Altschul et al., 1990) analysis on the 16S rRNA gene sequences, and the previously 235 identified rumen bacterium YS2 16S rRNA gene (accession number AF544207). To check for 
236 chloroplast contamination, a BLASTn analysis was performed on the 16S rRNA gene sequences,

237 identified as Cyanobacteria YS2, and the 16S rRNA gene sequence from Eucalyptus grandis

238 chloroplasts (accession number HM347959.1, Paiva et al., 2011).

239 Leaf collection method

240 Eucalypts respond to severe defoliation by producing abundant epicormic growth, which is 241 ontogenetically more similar to juvenile than to typical adult foliage. In the case of E. viminalis, 242 epicormic foliage accounted for the majority of foliage available to koalas in 2013 . To assess the 243 relative nutritional composition of the two-eucalypt species at Cape Otway, we collected epicormic 244 and adult E. viminalis $(\mathrm{n}=16)$ and E. obliqua $(\mathrm{n}=11)$ leaves from trees koalas were located in during 245 September 2013. Leaves were placed into labelled zip-lock bags and frozen at $-20^{\circ} \mathrm{C}$ until 246 processing. They were subsequently freeze-dried and ground to pass a $1 \mathrm{~mm}$ screen using a CT 247193 Cyclotec $^{\text {TM }}$ Sample Mill (Foss, Mulgrave, Victoria, Australia).

248

249

250

251

252

253

254

255

256

257

258

259

260

261

262

263

264

\section{Diet composition}

The chemical composition of cuticular wax often differs among the foliage of different species of higher plants. Due to their ability to traverse the gastrointestinal tract relatively intact, $n$-alkanes are the most frequently used wax marker in diet composition studies (Dove and Mayes, 2005). Therefore, we used the $n$-alkane protocol described by Dove and Mayes (2005) to estimate diet composition. Analysis was performed on an Agilent 7890A gas chromatograph coupled with an Agilent 5975C MSD (Agilent Technologies Pty Ltd, Mulgrave, VIC, Australia; details provided in the supplementary information). To estimate koala diet composition, $6 n$-alkane peaks $\left(\mathrm{C}_{23}, \mathrm{C}_{25}\right.$, $\mathrm{C}_{27}, \mathrm{C}_{28}, \mathrm{C}_{29}$ and $\left.\mathrm{C}_{31}\right)$ were identified and quantified in leaf $(\mathrm{n}=4$ samples per species $)$ and faecal samples using the Agilent MSD Chemstation v E.02.02 software package (Agilent Technologies Pty Ltd). Estimates of diet composition were determined following the calculations protocol described by Dove and Mayes (2005).

\section{Diet quality}

Nitrogen, tannins and fibre are key determinants of herbivore diet quality. In the common brushtail possum (Trichosurus vulpecula), a generalist marsupial folivore that also eats Eucalyptus, a measure of available N (AvailN, DeGabriel et al., 2009b) determined using an in vitro digestion assay, has successfully predicted reproductive success of free-living individuals (DeGabriel et al., 
265 2009a). We implemented the assay described by DeGabriel et al. (2008), which returns the 266 following measures of foliar quality: in vitro dry matter digestibility (DMD), total nitrogen 267 concentration $(\mathrm{N})$ and available, or digestible, $\mathrm{N}$ (AvailN). We analysed both epicormic (E. 268 viminalis $\mathrm{n}=9$ and E. obliqua $\mathrm{n}=5)$ and adult (E. viminalis $\mathrm{n}=7$ and E. obliqua $\mathrm{n}=6$ ) leaf samples. 269 Total $\mathrm{N}$ concentrations for leaf material and their residues after digestion, were measured by a 270 combustion method based on the Dumas method (Leco Corporation 2003) using the Leco C/N 271 analyser (Leco Tru Mac ${ }^{\circledR}$ Corporation, Michigan, USA). Analysis of the in vitro digestion data 272 was conducted using PRIMER v 7.0.13 and PERMANOVA+ to assess overall differences in 273 quality between the two diets, for assessment of the differences within and between diet variables 274 including mean total N, mean DMD, mean N digestibility (Ndig) and mean AvailN, the Students $275 t$-test was used.

276 Comparison of potential functional differences between Parabacteroides and Ruminococcaceae 277 genomes

278 The two dominant groups of bacteria displaying the largest change in relative abundance between

279 koala diet groups were the genus Parabacteroides and family Ruminococcaceae (see results). To 280 give an indication of the potential functional changes in the microbial community, we accessed 281 complete or draft linear genomes (at least 30× coverage) of 35 bacteria from Parabacteroides and 28245 from Ruminococcaceae (see supplementary information Table S5) with the "Genome Browser" 283 from the Microbial Genome and Metagenome Data Analysis pipeline of the Department of Energy 284 Joint Genome Institute (DOE JGI) site (https://img.jgi.doe.gov/cgi-bin/m/main.cgi). We assessed 285 the relationship between the 16S rRNA gene sequences, from the previously identified 286 Parabacteroides and Ruminococcaceae genomes and those isolated from the koalas' 287 microbiomes. We found that the koala 16S rRNA gene sequences were dispersed throughout the 288 constructed Parabacteroides and Ruminococcaceae phylogenetic trees (see supplementary 289 information Fig. S8 \& S9). The genomes were analysed for the presence of genes encoding enzymes and 290 transporters and grouped to glycoside hydrolase (GH) families according to the substrate specificities of 291 characterised enzymes, as stated in the Carbohydrate-Active Enzymes (CAZy) database (Cantarel et al., 292 2009; Berlemont and Martiny, 2015). GH families were classified as those targeting oligosaccharides, 293 starch and glycogen, cellulose, xylan, chitin, dextran, fructan, or other animal or plant polysaccharides; and 294 structural polysaccharides (i.e., cellulose, chitin, and xylan) as described by Berlemont and Martiny (2015). 295 Next, genomes were classified according to their potential for oligosaccharide and polysaccharide 
296 processing. Potential degraders of these substrates were defined as bacteria having at least one gene

297 targeting one of these specific substrates.

\section{Results}

299 Bacterial community rRNA gene sequencing

300 A total of $33,102,252$ reads with $31,832,423$ remaining post-sequence quality control were 301 obtained from the 32 samples sequenced (see supplementary information, Tables S1 \& S2a).

302 Faecal microbial communities of koalas eating E. viminalis were significantly less diverse 303 (Shannon $4.60 \pm 0.41$ vs $5.30 \pm 0.25$; ANOVA P=0.001) and less taxonomically rich (Chao 8,100 $304 \pm 2652$ vs $10,313 \pm 2079$; ANOVA $\mathrm{P}=0.001$ ) than those of koalas eating E. obliqua (see 305 supplementary information, Table S2b). Collection year was determined to be significant (Chao $306 \mathrm{P}=8.68 \mathrm{e}-13$ vs. Shannon $\mathrm{P}=0.121$ ), suggesting that evenness did not change between collection 307 years, but that richness in 2015 was higher than in 2013. We assessed whether differences between 308 the faecal bacterial communities associated with the two diets were driven by community structure, 309 i.e. differences in relative abundance of taxa (weighted UniFrac, Lozupone et al., 2011), or by 310 altered presence / absence of microbial taxa (unweighted UniFrac) using the 16S rRNA genes 311 retained after removal of chimeric sequences. These $\beta$-diversity matrices assess the extent of 312 branch sharing on a master phylogenetic tree, weighting branches by the relative abundance of 313 taxa (Lozupone et al., 2011). The UniFrac PCA biplots revealed a clear separation based on the

314 PC1 and PC2 axes of the weighted scatter plot, explaining 63\% of the total variation between the 315 two combined collections (Fig. 1a), reinforcing the observations from T-RFLP analysis (see 316 supplementary information Fig. S3). The unweighted scatter explained $18 \%$ of the total variation 317 (Fig. 1b). PERMANOVA analysis indicated no influence of collection year on the weighted 318 UniFrac data (Pseudo $F_{l}=0.44$, PERMANOVA P=0.81), but a significant influence was detected 319 when we analysed the unweighted UniFrac data (Pseudo $F_{l}=1.76$, PERMANOVA P=0.01). When 320 we analysed diet $\times$ collection years an influence was detected on community structure (weighted 321 UniFrac, Pseudo $F_{2}=10.07$, PERMANOVA P=0.0001) and presence / absence of microbial taxa 322 (unweighted UniFrac, Pseudo $F_{2}=2.98$, PERMANOVA P=0.0001).

323 PERMANOVA assessment of the weighted and unweighted UniFrac matrices from individual 324 collection years (2013 and 2015), indicated that diet was a strong driver of both microbial 325 community structure (relative abundance), and of microbial presence / absence during 2013 
326 (weighted UniFrac, Pseudo $F_{l}=5.88$, PERMANOVA P=0.0001; unweighted UniFrac, Pseudo $327 F_{l}=1.89$, PERMANOVA P=0.0001). The influence of diet was also significant in 2015 (weighted 328 UniFrac, Pseudo $F_{l}=8.89$, PERMANOVA P=0.0001; unweighted UniFrac, Pseudo $F_{l}=2.76$, 329 PERMANOVA P=0.0001). PCoA analysis of the weighted and unweighted UniFrac matrices for 330 the 2013 koala collection, showed a clear separation linked to diet, with $75 \%$ of total variation 331 explained by PC1 (weighted UniFrac, Fig. 2a), while the unweighted UniFrac matrix also indicated

332

333

334

335

336

337

338

339

340

341

342

343

344

345

346

347

348

349

350

351

352

353

354

355 an influence of diet, although the two axes explained less (30\%) of the total variation (Fig. $2 b$ ). Overall the separation between the microbiomes of the E. viminalis and E. obliqua koalas was smaller in 2015 than 2013 (average pairwise Unifac distance between groups \pm standard deviation, weighted: $2013=0.481 \pm 0.082,2015=0.377 \pm 0.075$; unweighted: $2013=0.756 \pm 0.072,2015=$ $0.666 \pm 0.073$ ). This was reflected in the 2015 PCoA analysis (Fig. 3a \& 3b). PC1 and PC2 of the weighted and unweighted PCoAs explained 65 and 18\% of the total variation, respectively. Similar patterns were observed during T-RFLP analysis of the two collection years (see supplementary information Fig. S4a \& S4b).

Analysis of phylum-level OTUs indicated a separation between diets for both 2013 and 2015 collections (2013, Pseudo $F_{l}=65.47$, PERMANOVA $\mathrm{P}=0.0001 ; 2015$ Pseudo $F_{l}=24.95$, PERMANOVA P $<0.0001$ respectively). In 2013, there was an almost three-fold increase in relative abundance of Bacteroidetes in E. viminalis koalas, compared with an almost three-fold increase in relative abundance of Firmicutes in the E. obliqua koalas (Table 1). The same pattern in relative abundance was observed in the 2015 koala faecal microbiomes, although to a lesser extent (Table 1). There were also significant differences in the relative abundance of some phyla between collection years. For example, the phylum Synergistetes was more abundant in the faecal microbiomes of koalas eating both E. viminalis and E. obliqua in 2015 (Table 1). Cyanobacteria increased in relative abundance in the 2015 E. viminalis faecal microbiomes by $15 \times$ that of the $E$. viminalis 2013 faecal microbiomes, where E. obliqua faecal microbiomes had an 75\% increase in relative abundance of Cyanobacteria (Table 1). Interestingly, the difference in relative abundance of Cyanobacteria between the two diets reversed in 2015 (Table 1). BLASTn analysis of the sequences identified as Cyanobacteria order YS2 confirmed that they are Cyanobacterial sequences (between 91 and 96\% identity with the 16S rRNA gene from rumen bacterium YS2 accession number AF544207) and not chloroplast contamination (between 74 and $88 \%$ identity with the $16 \mathrm{~S}$ 
356 rRNA gene from Eucalyptus grandis chloroplast genome accession number HM347959.1 (Paiva 357 et al., 2011).

358 Analysis of genus-level OTUs also revealed a significant separation between koala diets in 2013 359 (Pseudo $F_{l}=56.08$, PERMANOVA P=0.001; see supplementary information Fig. S5a) and 2015 360 (Pseudo $F_{l}=17.04$, PERMANOVA P=0.001; see supplementary information Fig. S5b). In 2013, 361 there were notable diet-associated differences in relative microbial abundance, in particular, the $E$. 362 viminalis koala faecal microbiomes were dominated by Parabacteroides (52 \pm 4 ; Fig. 4a; see 363 supplementary information, Table S3), while the faecal microbiomes of E. obliqua koalas were 364 dominated by an unknown genus from the family Ruminococcaceae (45 \pm 1 ; Fig. 4a; see 365 Supplementary information, Table S3). BLASTn database searches (Altschul et al., 1990) were 366 unable to improve taxonomic resolution.

367 In 2015, the genus dominating faecal microbiomes of koalas eating E. viminalis was Bacteroides 368 (25 \pm 5 ; Fig. 4b; see supplementary information, Table S3). The relative abundance was $3.5 \times$ that seen in the 2013 E. viminalis koala faecal microbiomes, while the relative abundance of

370 Parabacteroides was $42 \%$ lower in the 2015 E. viminalis koala faecal microbiomes compared with 371 the 2013 (Fig. 4a \& 4b; see supplementary information, Table S3). In both 2013 and 2015, E.

372 obliqua faecal microbiome communities were dominated by the family Ruminococcaceae $(31 \pm$ 373 4; Fig. 3a \& 3b; see Supplementary information, Table S4). However, the 2015 E. obliqua faecal 374 bacterial communities had almost four $\times$ the Parabacteroides $(19 \pm 4)$, and half the Bacteroides 375 relative abundances compared with 2013 E. obliqua faecal microbiome communities (Fig. 4a \& 376 4b; see Supplementary information, Table S3). Other genera represented in both 2013 and 2015 377 faecal microbiomes at relative abundances from 3 to 13\% include Acidaminococcus, Akkermansia, 378 Coprobacillus, Clostridium, Oscillospira and Ruminococcus, (Fig. 4a \& 4b; see supplementary 379 information, Table S3). Relative abundance of these OTUs showed differences within and / or 380 between the two collection years and diet types, while the remaining genera did not show 381 significant differences (Fig. 4a \& 4b; see supplementary information Table S3).

382 Functional differences between previously identified and publicly available Parabacteroides and 383 Ruminococcaceae genomes

384 Due to the dominance and significant changes in relative abundance of Parabacteroides and 385 Ruminococcaceae we analysed 35 previously identified and publicly available, Parabacteroides 
386

387

388

389

390

391

392

393

394

395

396

397

398

399

400

401

402

403

404

405

406

407

408

409

410

411

412

413

414

415

and 45 Ruminococcaceae genomes and identified glycoside hydrolase (GH) families involved in the degradation of plant cell walls, starch and other components ranging from easily degraded to recalcitrant (see supplementary information Fig. S7). In general, we found that the Parabacteroides genomes (associated with E. viminalis diets) possessed more genes for oligosaccharide degradation than Ruminococcaceae genomes, while Ruminococcaceae genomes (more strongly associated with E. obliqua diets) possessed up to five $\times$ the number of enzymatic genes targeting the degradation of recalcitrant cellulose (see supplementary information Fig. S7). Parabacteroides genomes also have more genes from the GH67 and GH85 families, which are involved in the degradation of xylan and chitin, respectively. Interestingly, Parabacteroides genomes potentially have more genes associated with tannin degradation than Ruminococcaceae (see supplementary information Fig. S7). ATP-binding cassette (ABC) and phosphotransferase system (PTS) transporter genes were more common in Ruminococcaceae (average of 134 ABC and 18 PTS per genome, see supplementary information, Table S5) than in Parabacteroides (82 ABC and 6 PTS, see supplementary information, Table S5).

\section{Diet composition}

Because of the strong association observed between diet and microbiome, we analysed koala faecal pellets to confirm our expectation of diet composition (based upon koala location and tree occupancy), using methods established to estimate composition of diets using the $n$-alkanes that occur in the leaf cuticle of all plants as markers (Dove and Mayes, 2005).

Analysis of $n$-alkane markers in E. viminalis and E. obliqua leaf material showed significantly different profiles between species, with $E$. obliqua cuticle dominated by the $\mathrm{C}_{27}$ chain-length $n$ alkane ( $78 \pm 3$ of total alkanes) and $E$. viminalis dominated by $\mathrm{C}_{29}(91 \pm 3)$. The relative abundance of the $\mathrm{C}_{25}$ alkane was also 7 times greater in E. obliqua than E. viminalis. Koala faecal alkane profiles clearly separated our two diet categories and largely confirmed our expectations about diet composition based on tree canopy composition at koala pellet collection sites. In 2013, the $n$ alkane method estimated that $8 / 9$ "E. viminalis" koalas included $>80 \%$ E. viminalis in their diets, and 5/5 "E. obliqua" koalas included $>80 \%$ E. obliqua (Fig. 5a). In 2015, 8/8 "E. viminalis" diets were estimated to contain $>70 \%$ E. viminalis, and 6/7 "E. obliqua" diets $>50 \%$ E. obliqua (Fig. $5 b)$. 
417 Nutritional analysis of E. viminalis and E. obliqua epicormic and adult leaves indicated that overall

418 E. viminalis provided koalas with foliage of higher nutritional quality (i.e. greater in vitro dry 419 matter digestibility (DMD), available (or digestible) N (AvailN) and total foliar N concentrations; 420 Pseudo $F_{l}=36.43$ and PERMANOVA P=0.001) compared with E. obliqua foliage (Table 2). The 421 lower AvailN of E. obliqua is due to both lower total N concentrations and lower N digestibility 422 (NDig), the latter indicating a stronger effect of anti-nutritional tannins (DeGabriel et al., 2008). 423 We confirmed that adult E. viminalis foliage contained less recalcitrant fibre (i.e. hemicellulose, 424 lignin and cellulose) than adult E. obliqua foliage, using the neutral detergent fibre (NDF) assay 425 (Van Soest et al., 1991; $\mathrm{P}=0.003$, Table 2).

\section{Discussion}

427 Dramatic diet changes in humans e.g. switching from carnivorous to herbivorous diets, can 428 profoundly affect the microbial community structure in the gut (Ley et al., 2008a,b; David et al., 2014). Here we show that equally dramatic effects can be produced, even within a continuous animal population, through a change in consumption from one to another congeneric tree species. Bacterial communities associated with each of the two diets differed primarily in the relative abundance of two phyla, Bacteroidetes and Firmicutes, rather than in presence and absence of abundant taxa similar to changes seen in humans when undergoing major diet change (David et al., 2014). Alpha diversity was significantly lower in terms of Shannon diversity and richness (Chao1) in koalas eating the nutritionally superior E. viminalis. Low microbial richness has been convincingly linked to higher feed efficiency in ruminants (Shabat et al., 2016). In our study, lower diversity and richness is likely to be associated with a greater energy harvest from E. viminalis compared to E. obliqua. Efficient microbiomes are often less complex but more specialised, which creates higher availability of ecosystem goods, such as energy resources, to the host (De Groot et al., 2002; Shabat et al., 2016). In addition, the general rearrangement of taxa within the less diverse phyla Bacteroidetes and the more diverse Firmicutes (Parks et al., 2018) in response to change in diet, could also contribute to lower richness and diversity in koalas eating E. viminalis compared to E. obliqua. Southern Australian koalas from E. viminalis forests and woodlands are unusual in specialising on a single eucalypt species, but such specialisation over many generations may have resulted in an equally unusual, specialised microbiome. 
446 Associations between diet and the relative abundance of Bacteroidetes and Firmicutes have been

447 linked to the functional potential of the gut microbiome in humans and animals (Muegge et al.,

448 2011). For instance, Bacteroidetes genomes encode GH enzymes targeting a wide variety of 449 relatively easily degraded plant components including non-cellulosic polysaccharides, 450 oligosaccharides and glycogen (Hooper et al., 2002). In contrast, Firmicutes genomes encode GHs 451 targeting cellulases and xylanases that would be beneficial in an environment dominated by 452 recalcitrant fibre (Ben David et al., 2015). High prevalence of Firmicutes and a diminished relative 453 abundance of Bacteroidetes has been associated with adjustments of the microbiome to increased 454 fibre intake and reduced protein consumption during transitions from animal to plant diets (David 455 et al., 2014; Ben David et al., 2015). Similarly, shifts in the Bacteroidetes: Firmicutes ratio in 456 koalas eating E. obliqua could be due to the lower available protein and higher fibre content of $E$. 457 obliqua as compared to E. viminalis.

458 We suggest several further potential functional differences in microbiomes associated with diet in 459 koalas. Koalas eating E. viminalis hosted bacterial communities dominated by Parabacteroides, 460 the genomes of which usually encode multiple enzymes that sense, bind and metabolise a variety 461 of oligosaccharides (Mahowald, 2010). These may represent a larger fraction of leaf material in $E$. 462 viminalis foliage, which is $50 \%$ more digestible in-vitro than E. obliqua, which have more 463 recalcitrant cell wall components compared with adult leaf foliage from E. viminalis (48 and 38\% 464 respectively). However, caution is required in interpretation of the NDF results as tannins are 465 known to inflate NDF measurements, leading to an overestimation of fibre (Makkar et al., 1995). 466 The microbiome associated with E. obliqua was dominated by the family Ruminococcaceae. 467 Ruminococcaceae have smaller genomes than Parabacteroides, with fewer glycan-degrading 468 enzymes, and are suited to the degradation of more varied dietary carbohydrates (Biddle et al., 469 2013; Ben David et al., 2015). They have more ABC and PTS transporters that may provide a 470 competitive advantage over Parabacteroides, by facilitating faster bacterial uptake of sugars 471 (Biddle et al., 2013). The gut lumen of koalas eating E. obliqua would have higher concentrations 472 of tannins and recalcitrant fibre, so while the observation of greater cellulose-degrading 473 functionality associated with Ruminococcaceae is expected, the potentially greater tannin474 degrading functionality of Parabacteroides is more surprising. Little is known of qualitative 475 variation in tannin composition among eucalypt species. 
476 Cyanobacteria (the order YS2) were observed at high relative abundance (up to 30\%) in many of 477 our samples, particularly in 2015 where the average relative abundance for koalas eating $E$. 478 viminalis was $15 \%$. Such high relative abundances are unprecedented in gastrointestinal 479 microbiomes. Ley et al., (2008a) reported an average relative abundance of 1\% across mammals 480 generally, although higher relative abundances have been reported e.g. 5\% from a domestic cow 481 (Bos taurus) and a capybara (Hydrochoerus hydrochaeris, Ley et al., 2008a), 6.7\% and 9\% for 482 individual koalas (Soo et al., 2014; Shiffman et al., 2017), and 4.7\% and 4.5\% for domestic rabbits 483 and American pikas (Oryctolagus cuniculus, Zeng et al., 2015; Ochotona princeps, Kohl et al., 484 2017). Non-photosynthetic Cyanobacteria, now placed within the candidate phylum Melainabacteria (Di Rienzi et al., 2013; Soo et al., 2014), have only recently been recognized in gastrointestinal microbiomes, they are obligate anaerobic fermenters and syntrophic hydrogen producers which may benefit the host by the synthesis of vitamins B and K (Di Rienzi et al., 2013), and which are enriched in Kyoto Encyclopedia of Genes and Genomes (KEGG) Ortholog groups specific to amino acid metabolism, relative to other cyanobacteria (Harel et al., 2015).

The relatively low relative abundance of Proteobacteria (1\%) in koala faeces from Cape Otway is less than that reported from faecal samples across mammals and herbivores generally $(8.8 \%$ and 5\%, Ley et al., 2008a; Nelson et al., 2013) and lower than detected in the folivorous two-and threetoed sloths (Choloepus hoffmanni and Bradypus variegatus, 60\%; Dill-McFarland et al., 2015). It is also substantially lower than that observed in zoo koalas by Shiffman et al., (2017) and Barker et al., (2013; 15\% and 2 - 9\% respectively), but consistent with the observations of Alfano et al., (2015).

Low-relative abundance microbes can also be functionally important in the gut microbiome (Qin et al., 2010). Synergistes is an anaerobic fermenter of some amino acids (Allison et al., 2015), and by fermenting the toxic amino acid, mimosine, in the forage legume, Leucaena leucocephala, protects ruminant hosts from toxicosis (Allison et al., 1992). Synergistes is also a member of a consortium that can protect sheep from pyrrolizidine alkaloid toxicosis (Lodge-Ivey et al., 2005; Rattray and Craig, 2007) and can anaerobically degrade fluoracetate (Davis et al., 2012). Shiffman et al., (2017) suggested that Synergistaceae may play as-yet unknown roles in addition to those above in koalas, including the degradation of plant toxins from Eucalyptus. On the basis of its metabolic potential and the relatively high abundance of Synergistaceae that they observed in 
506 koalas relative to most other gut ecosystems, they also identified it as the most likely core 507 specialised member of the koala microbiota. However, this phylum occurred at lower 508 concentrations in faecal microbiomes from Cape Otway, particularly in 2013 where mean 509 abundances were as low as $0.0004 \%$ (E. obliqua). This suggests a key role of Synergistaceae in 510 allowing the koala to subsist on Eucalyptus, as proposed by Shiffman et al., (2017), is either not 511 essential at least for some koala diets, or can be filled by other bacteria.

512 Another bacterial population previously identified by Shiffman et al., (2017) as discriminating the 513 koala microbiota from the wombat microbiota is the family S24-7 (phylum Bacteroidetes), which 514 they observed at a mean relative abundance greater than 10\% in zoo koalas, and which they linked 515 to dietary specialisation in Eucalyptus. Ormerod et al., (2016) detected two trophic guilds $(\alpha-$ 516 glucan and plant glucan) among $S 24-7$ population genomes isolated from a koala; these genomes 517 were remarkable for their large size relative to other S24-7. Shiffman et al. (2017) also detected a 518 full suite of ureolysis genes in an $\$ 24-7$ genome accounting for $\sim 8 \%$ of the faecal microbial 519 community. However, S24-7 was not found amongst wild Cape Otway koalas, echoing the 520 findings of Barker et al., (2013), who detected S24-7 at only low abundance (0.07\%) in the caeca 521 of two wild koalas, but not in their faecal pellets.

522 In common with other culture-independent investigations of the koala microbime (Alfano et al., 523 2015; Barker et al., 2013; Shiffman et al., 2017), our study also found that tannin-protein complex 524 degrading bacteria (Enterobacteriaceae, Pasteurellaceae and Streptococcaceae) previously 525 cultured by Osawa and colleagues (Osawa 1990; Osawa 1992; Osawa et al., 1993; Osawa et al., 526 1995) were rare and occurred at low abundance (4 and 1\% for 2013 and 2015 collections 527 respectively) in wild koala faeces.

528 In addition to community differences associated with diet, differences were also apparent between 529 the two collection years. For instance, Bacteroides relative abundance was three $\times$ greater in $E$. 530 viminalis communities in 2015 than in 2013, while Parabacteroides relative abundance was lower 531 in E. viminalis but $47 \%$ greater in E. obliqua diets in 2015 compared to 2013 . We speculate that 532 these differences might be explained by differences in food availability, leaf type and/or nutritional 533 status for E. viminalis koalas between 2013 and 2015. Therefore, observed differences in microbial 534 relative abundance between collection years might be explained by a return to a "normal diet" after 535 the peak of overbrowsing associated with peak koala population densities; this would be consistent 
536 with the diet composition analysis, which suggested that all koalas were eating E. viminalis to

537 some extent in 2015. Periodic fasting through dietary restriction or seasonal hibernation has been

538 found to alter the microbial community structure in mammals (Clarke et al., 2012). In particular, 539 other studies have observed increases in acetate-producing bacteria including Akkermansia 540 muciniphila during periods of fasting, and suggested that under these circumstances, host-derived 541 polysaccharides such as mucins are used as a substrate to produce short-chain fatty acids that 542 support the host (Carey et al., 2013; Derrien et al., 2008; Sonoyama et al., 2009). The relative 543 abundance of Akkermansia was notably elevated (between 1 and 13\%) in some of the koalas eating 544 E. viminalis in 2013, and this may indicate that these individuals were experiencing a shortage of 545 food. However, despite the differences in microbial relative abundance associated with collection 546 period, the gut microbial communities associated with the two different diets remained distinct 547 throughout.

548 Here we have shown that even a seemingly subtle dietary change can modulate the microbiome of 549 a specialist herbivore. Based on our results, we postulate that diet preferences and the availability 550 of resources will substantially impact the structure of gut microbial communities of koalas more 551 widely, with consequences for the efficiency of digestion of their complex diet. As strong regional 552 differences have been observed in the diet composition of koalas across their geographic range, 553 translocated and released koalas (e.g. after overpopulation, habitat degradation, or rehabilitation) 554 and those treated with antibiotics may not have the same microbiome as natural populations. Thus, 555 a priority area of research should be to determine whether the therapeutic or prophylactic alteration 556 of koala microbiomes can assist management and welfare outcomes for koalas facing enforced 557 dietary change. New conservation and management strategies could include the development of 558 targeted inoculations, thereby facilitating an increased dietary breadth for koalas, as demonstrated 559 by Kohl et al., (2014d) and Kohl et al., (2015) in the specialist desert woodrat.

\section{Conclusion}

561 We show that diet differences, such as a change in consumption from one to another congeneric 562 tree species, can profoundly affect the gut microbiome of a specialist folivorous mammal, even 563 amongst individuals within a single contiguous population.

564 
566

567

568

569

570

571

572

573

574

575

576

577

578

579

580

581

582

583

584

585

586

587

588

589

590

591

592

593

594

595

596

597

598

599

600

601

602

603

604

Acknowledgements

The authors would like to acknowledge and thank the following people: Desley Whisson, Scott

Bevins, Jack Pascoe, Lizzie Corke, Shayne Neal and the Conservation Ecology Centre at Cape

Otway, Manuel Delgado Baquerizo and Jasmine Grinyer. The technical staff at HIE for running samples including T-RFLP and dietary analysis including GC-MS and C:N analysis. The staff of the WSU NGS centre for following and optimising the V4 original Earth Microbiome protocol.

Supplementary information is available at PeerJ's website.

\section{References}

Alfano, N., Courtiol, A., Vielgrader, H., Timms, P., Roca, A.L., and Greenwood, A.D. (2015) Variation in koala microbiomes within and between individuals: effect of body region and captivity status. Sci. Rep. $\mathbf{5}$.

Allison, M., MacGregor, B., and Stahl, D. (2015) Synergistes. In Bergey’s Manual ${ }^{\circledR}$ of Systematics of Archaea and Bacteria Bacteriology: Springer, pp. 1-3.

Allison, M.J., Mayberry, W.R., Mcsweeney, C.S., and Stahl, D.A. (1992) Synergistes jonesii, gen. nov., sp. nov.: a rumen bacterium that degrades toxic pyridinediols. Syst. Appl. Microbiol. 15: 522-529.

Altschul, S.F., Gish, W., Miller, W., Myers, E.W., and Lipman, D.J. (1990) Basic local alignment search tool. J. Mol. Biol. 215: 403-410.

Amato KR, Yeoman CJ, Kent A, Righini N, Carbonero F, Estrada A, Gaskins HR, Stumpf RM, Yildirim S, Torralba M, Gillis M. (2013) Habitat degradation impacts black howler monkey (Alouatta pigra) gastrointestinal microbiomes. ISME J. 7: 1344-1353.

Anderson, M.J. (2001) A new method for non-parametric multivariate analysis of variance. Austral Ecol. 26: 32-46.

Barelli, C., Albanese, D., Donati, C., Pindo, M., Dallago, C., Rovero, F., Cavalieri, D., Tuohy, K.M., Hauffe, H.C. and De Filippo, C. (2015) Habitat fragmentation is associated to gut microbiota diversity of an endangered primate: implications for conservation. Sci. Rep. $\mathbf{5}$ : 14862.

Barker, C.J., Gillett, A., Polkinghorne, A., and Timms, P. (2013) Investigation of the koala (Phascolarctos cinereus) hindgut microbiome via 16S pyrosequencing. Vet. Microbiol. 167: 554-564.

Ben David Y, Dassa B, Borovok I, Lamed R, Koropatkin NM, Martens EC, White BA, Bernalier-Donadille A, Duncan SH, Flint HJ, Bayer EA. (2015) Ruminococcal cellulosome systems from rumen to human. Environ. Microbiol. 17: 3407-3426.

Biddle, A., Stewart, L., Blanchard, J., and Leschine, S. (2013) Untangling the Genetic Basis of Fibrolytic Specialization by Lachnospiraceae and Ruminococcaceae in Diverse Gut Communities. Diversity 5: 627-640.

Caporaso, J.G., Bittinger, K., Bushman, F.D., DeSantis, T.Z., Andersen, G.L., and Knight, R. (2010a) PyNAST: a flexible tool for aligning sequences to a template alignment. Bioinformatics 26: 266-267. 
605

606

607

608

609

610

611

612

613

614

615

616

617

618

619

620

621

622

623

624

625

626

627

628

629

630

631

632

633

634

635

636

637

638

639

640

641

642

643

644

645

646

647

648

Caporaso, J.G., Lauber, C.L., Walters, W.A., Berg-Lyons, D., Lozupone, C.A., Turnbaugh, P.J., Fierer, N. and Knight, R.. (2011a) Global patterns of 16S rRNA diversity at a depth of millions of sequences per sample. PNAS 108: 4516-4522.

Caporaso, J.G., Kuczynski, J., Stombaugh, J., Bittinger, K., Bushman, F.D., Costello, E.K., Fierer, N., Pena, A.G., Goodrich, J.K., Gordon, J.I. and Huttley, G.A. (2010b) QIIME allows analysis of high-throughput community sequencing data. Nat. Methods 7: 335-336.

Carey, H.V., Walters, W.A., and Knight, R. (2013) Seasonal restructuring of the ground squirrel gut microbiota over the annual hibernation cycle. Am. J. Physiol. Regul. Integr. Comp. Physiol. 304: R33-R42.

Clarke, K.R. (1993) Non-parametric multivariate analyses of changes in community structure. Aust. J. Ecol. 18: 117-143.

Clarke, S.F., Murphy, E.F., Nilaweera, K., Ross, P.R., Shanahan, F., O’Toole, P.W., and Cotter, P.D. (2012) The gut microbiota and its relationship to diet and obesity: new insights. Gut microbes 3: 186-202.

Cork, S.J., and Warner, A. (1983a) The passage of digesta markers through the gut of a folivorous marsupial, the koalaPhascolarctos cinereus. J. Comp. Physiol. 152: 43-51.

David, L.A., Maurice, C.F., Carmody, R.N., Gootenberg, D.B., Button, J.E., Wolfe, B.E., Ling, A.V., Devlin, A.S., Varma, Y., Fischbach, M.A. and Biddinger, S.B. (2014) Diet rapidly and reproducibly alters the human gut microbiome. Nat. 505: 559-563.

Davis, C.K., Webb, R.I., Sly, L.I., Denman, S.E., and McSweeney, C.S. (2012) Isolation and survey of novel fluoroacetate-degrading bacteria belonging to the phylum Synergistetes. FEMS Microbiol. Ecol. 80: 671-684.

De Groot, R.S., Wilson, M.A., and Boumans, R.M. (2002) A typology for the classification, description and valuation of ecosystem functions, goods and services. Ecol. Econ. 41: 393408.

DeGabriel, J.L., Wallis, I.R., Moore, B.D., and Foley, W.J. (2008) A simple, integrative assay to quantify nutritional quality of browses for herbivores. Oecol. 156: 107-116.

DeGabriel, J.L., Moore, B.D., Foley, W.J., and Johnson, C.N. (2009a) The effects of plant defensive chemistry on nutrient availability predict reproductive success in a mammal. Ecol. 90: 711-719.

DeGabriel, J.L., Moore, B.D., Shipley, L.A., Krockenberger, A.K., Wallis, I.R., Johnson, C.N., and Foley, W.J. (2009b) Inter-population differences in the tolerance of a marsupial folivore to plant secondary metabolites. Oecol. 161: 539-548.

Derrien, M., Collado, M.C., Ben-Amor, K., Salminen, S., and de Vos, W.M. (2008) The Mucin degrader Akkermansia muciniphila is an abundant resident of the human intestinal tract. Appl. Environ. Microbiol. 74: 1646-1648.

DeSantis, T.Z., Hugenholtz, P., Larsen, N., Rojas, M., Brodie, E.L., Keller, K., Huber, T., Dalevi, D., Hu, P. and Andersen, G.L. (2006) Greengenes, a chimera-checked 16S rRNA gene database and workbench compatible with ARB. Appl. Eenviron. Microbiol. 72: 50695072.

Di Rienzi, S.C., Sharon, I., Wrighton, K.C., Koren, O., Hug, L.A., Thomas, B.C., Goodrich, J.K., Bell, J.T., Spector, T.D., Banfield, J.F. and Ley, R.E. (2013) The human gut and groundwater harbor non-photosynthetic bacteria belonging to a new candidate phylum sibling to Cyanobacteria. Elife 2: e01102. 
649

650

651

652

653

654

655

656

657

658

659

660

661

662

663

664

665

666

667

668

669

670

671

672

673

674

675

676

677

678

679

680

681

682

683

684

685

686

687

688

689

690

691

692

693

Dill-McFarland, K.A., Weimer, P.J., Pauli, J.N., Peery, M.Z., and Suen, G. (2015) Diet specialization selects for an unusual and simplified gut microbiota in two- and three-toed sloths. Environ. Microbiol. 18: 1391-1402.

Dove, H., and Mayes, R. (2005) Using n-alkanes and other plant wax components to estimate intake, digestibility and diet composition of grazing/browsing sheep and goats. Small Rumin. Res. 59: 123-139.

Edgar, R.C. (2010) Search and clustering orders of magnitude faster than BLAST. Bioinformatics. 26: 2460-2461.

Eschler, B., Pass, D., Willis, R., and Foley, W. (2000) Distribution of foliar formylated phloroglucinol derivatives amongst Eucalyptus species. Biochem. Syst. Ecol. 28: 813-824.

Flint, H.J., and Bayer, E.A. (2008b) Plant cell wall breakdown by anaerobic microorganisms from the Mammalian digestive tract. Ann. N. Y. Acad. Sci. 1125: 280-288.

Foley, W.J., and Cork, S.J. (1992) Use of fibrous diets by small herbivores: how far can the rules be 'bent'? Trends Ecol. Evolut. 7: 159-162.

Foley, W.J., Lassak, E.V. and Brophy, J. (1987) Digestion and absorption of Eucalytpus essential oils in greater glider (Petauroides volans) and brushtail possum (Trichosurus vulpecula). J. Chem. Ecol. 13:2115-2130.

Godon, J.J., Morinière, J., Moletta, M., Gaillac, M., Bru, V., and Delgènes, J.P. (2005) Rarity associated with specific ecological niches in the bacterial world: the 'Synergistes' example. Environ. Microbiol. 7: 213-224.

Good, I.J. (1953) The population frequencies of species and the estimation of population parameters. Biometrika. 40: 237-264.

Haas, B.J., Gevers, D., Earl, A.M., Feldgarden, M., Ward, D.V., Giannoukos, G., Ciulla, D., Tabbaa, D., Highlander, S.K., Sodergren, E. and Methé, B. (2011) Chimeric 16S rRNA sequence formation and detection in Sanger and 454-pyrosequenced PCR amplicons. Genome Res. 21: 494-504.

Harel, A., Karkar, S., Cheng, S., Falkowski, P.G., and Bhattacharya, D. (2015) Deciphering primordial cyanobacterial genome functions from protein network analysis. Curr. Biol. 25: 628-634.

Hooper, L.V., Midtvedt, T., and Gordon, J.I. (2002) How host-microbial interactions shape the nutrient environment of the mammalian intestine. Annu. Rev. Nutr. 22: 283-307.

Hume, I.D. (ed) (2005) Concepts of digestive efficiency. Enfield: Science Publishers Inc. Hume, I.D., Esson, C., (1993) Nutrients, antinutrients and leaf selection by captive koalas (Phascolarctos cinereus). Aust. J. Zool. 41: 379-392.

Knezevic, P., Aleksic, V., Simin, N., Svircev, E., Petrovic, A. and Mimica-Dukic, N. (2016) Antimicrobial activity of Eucalyptus camaldulensis essential oils and their interactions with conventional antimicrobial agents against multi-drug resistant Acinetobacter baumannii. J. Ethnopharmacol. 178: 125-136.

Kohl, K.D., and Dearing, M.D. (2012) Experience matters: prior exposure to plant toxins enhances diversity of gut microbes in herbivores. Ecol. Lett. 15: 1008-1015.

Kohl, K.D., Stengel, A., and Dearing, M.D. (2015) Inoculation of tannin-degrading bacteria into novel hosts increases performance on tannin-rich diets. Env.Microb. 18: 1720-1729.

Kohl, K.D., Varner, J., Wilkening, J.L., and Dearing, M.D. (2017) Gut microbial communities of American pikas (Ochotona princeps): Evidence for phylosymbiosis and adaptations to novel diets. J. Anim. Ecol. 87:323-330. 
694

695

696

697

698

699

700

701

702

703

704

705

706

707

708

709

710

711

712

713

714

715

716

717

718

719

720

721

722

723

724

725

726

727

728

729

730

731

732

733

734

735

736

737

738

739

Kohl, K.D., Weiss, R.B., Cox, J., Dale, C., and Denise Dearing, M. (2014d) Gut microbes of mammalian herbivores facilitate intake of plant toxins. Ecol. Lett. 17: 1238-1246.

Krockenberger, A.K., and Hume, I.D. (2007) A flexible digestive strategy accommodates the nutritional demands of reproduction in a free-living folivore, the Koala (Phascolarctos cinereus). Funct. Ecol. 21: 748-756.

Ley, R.E., Hamady, M., Lozupone, C., Turnbaugh, P.J., Ramey, R.R., Bircher, J.S., Schlegel, M.L., Tucker, T.A., Schrenzel, M.D., Knight, R. and Gordon, J.I. (2008a) Evolution of mammals and their gut microbes. Science 320: 1647-1651.

Ley, R.E., Lozupone, C.A., Hamady, M., Knight, R. and Gordon, J.I., (2008b) Worlds within worlds: evolution of the vertebrate gut microbiota. Nat. Rev. Microbiol.a 6: 776-788.

Lichtman, J.S., Sonnenburg, J.L., and Elias, J.E. (2015) Monitoring host responses to the gut microbiota. ISME J. 9: 1908-1915.

Lodge-Ivey, S., Rappe, M., Johnston, W., Bohlken, R., and Craig, A. (2005) Molecular analysis of a consortium of ruminal microbes that detoxify pyrrolizidine alkaloids. Can. J. Microbiol. 51: 455-465.

Lozupone, C., Lladser, M.E., Knights, D., Stombaugh, J., and Knight, R. (2011) UniFrac: an effective distance metric for microbial community comparison. ISME J. 5: 169-172.

Mahowald, M. (2010) Functional Genomic Examinations Of Interactions Between Common Members Of The Human Gut Microbiota. Washington University in St. Louis. Available from:

https://openscholarship.wustl.edu/cgi/viewcontent.cgi?referer=https://scholar.google.com.au/ \&httpsredir $=1 \&$ article $=1221 \&$ context $=$ etd [accessed on 20 October 2014].

Makkar, H., Borowy, N., Becker, K., and Degen, A. (1995) Some problems in fiber determination of a tannin-rich forage (Acacia saligna leaves) and their implications in in vivo studies. Anim. Feed Sci. Technol. 55: 67-76.

Marsh, K.J., Moore, B.D., Wallis, I.R., and Foley, W.J. (2014) Feeding rates of a mammalian browser confirm the predictions of a 'foodscape'model of its habitat. Oecol. 174: 873-882.

Marsh, K.J., Yin, B., Singh, I.P., Saraf, I., Choudhary, A., Au, J., Tucker, D.J. and Foley, W.J. (2015) From Leaf Metabolome to In Vivo Testing: Identifying Antifeedant Compounds for Ecological Studies of Marsupial Diets. J. Chem. Ecol. 41: 513-519.

Marsh, K.J., Kulheim, C., Blomberg, S.P., Thornhill, A.H., Miller, J.T., Wallis, I.R., Nicolle, D., Salminen, J.P. and Foley, W.J. (2017) Genus-wide variation in foliar polyphenolics in eucalypts. Phytochemistry 144: 197-207.

Martin, R.W. (1985a) Overbrowsing, and decline of a population of the koala, Phascolarctos cinereus, in Victoria. I. Food preference and food tree defoliation. Wildl. Res. 12: 355-365.

Miller, A.W., Kohl, K.D., and Dearing, M.D. (2014) The gastrointestinal tract of the whitethroated Woodrat (Neotoma albigula) harbors distinct consortia of oxalate-degrading bacteria. Appl. Environ. Microbiol. 80: 1595-1601.

Moore, B.D., and Foley, W.J. (2005b) Tree use by koalas in a chemically complex landscape. Nature 435: 488-490.

Moore, B.D., Wallis, I.R., Marsh, K.J., and Foley, W.J. (2004a) The role of nutrition in the conservation of the marsupial folivores of eucalypt forests. Mosman, NSW: Royal Zoological Society of New South Wales.

Moore, B.D., Wallis, I.R., Wood, J.T., and Foley, W.J. (2004c) Foliar nutrition, site quality, and temperature influence foliar chemistry of tallowood (Eucalyptus microcorys). Ecol. Monograph. 74: 553-568. 
740

Moore, B.D., Wallis, I.R., Palá-Paúl, J., Brophy, J.J., Willis, R.H., and Foley, W.J. (2004b) Antiherbivore chemistry of Eucalyptus cues and deterrents for marsupial folivores. J. Chem. Ecol. 30: 1743-1769.

Muegge BD, Kuczynski J, Knights D, Clemente JC, González A, Fontana L, Henrissat B, Knight R, Gordon JI. (2011) Diet drives convergence in gut microbiome functions across mammalian phylogeny and within humans. Science 332: 970-974.

Nelson, T.M., Rogers, T.L., and Brown, M.V. (2013) The gut bacterial community of mammals from marine and terrestrial habitats. PLoS One 8: e83655.

Ormerod KL, Wood DL, Lachner N, Gellatly SL, Daly JN, Parsons JD, Dal'Molin CG, Palfreyman RW, Nielsen LK, Cooper MA, Morrison M. (2016) Genomic characterization of the uncultured Bacteroidales family $S 24-7$ inhabiting the guts of homeothermic animals. Microbiome 4: 36.

Osawa, R. (1990) Formation of a clear zone on tannin-treated brain heart infusion agar by a Streptococcus sp. isolated from feces of koalas. Appl. Environ. Microbiol. 56: 829-831.

Osawa, R. (1992) Tannin-protein complex-degrading enterobacteria isolated from the alimentary tracts of koalas and a selective medium for their enumeration. Appl. Environ. Microbiol. 58: 1754-1759.

Osawa, R., Blanshard, W.H., and Ocallaghan, P.G. (1993) Microbiological studies of the intestinal microflora of the Koala, Phascolarctos cinereus. II. Pap, a special maternal feces consumed by Juvenile Koalas. Aust. J. Zool. 41: 611-620.

Osawa, R., Rainey, F., Fujisawa, T., Lang, E., Busse, H.J., Walsh, T.P., and Stackebrandt, E. (1995) Lonepinella koalarum gen. nov., sp. nov., a New Tannin-Protein Complex Degrading Bacterium. Syst. Appl. Microbiol. 18: 368-373.

Paiva, J.A., Prat, E., Vautrin, S., Santos, M.D., San-Clemente, H., Brommonschenkel, S., Fonseca, P.G., Grattapaglia, D., Song, X., Ammiraju, J.S. and Kudrna, D. (2011) Advancing Eucalyptus genomics: identification and sequencing of lignin biosynthesis genes from deepcoverage BAC libraries. BMC genomics 12: 137.

Parks, D.H., Chuvochina, M., Waite, D.W., Rinke, C., Skarshewski, A., Chaumeil, P.-A., and Hugenholtz, P. (2018) A proposal for a standardized bacterial taxonomy based on genome phylogeny. bioRxiv: 256800.

Provenza, F.D. (2006) Postingestive feedback as an elementary determinant of food preference and intake in ruminants. J. Range Manage. Arch. 48: 2-17.

Qin J, Li R, Raes J, Arumugam M, Burgdorf KS, Manichanh C, Nielsen T, Pons N, Levenez F, Yamada T, Mende DR. (2010) A human gut microbial gene catalogue established by metagenomic sequencing. Nature 464: 59-65.

R Development Core Team (2013) R: A language and environment for statistical computing. In $R$ Foundation for Statistical Computing (2013).

Rattray, R.M., and Craig, A.M. (2007) Molecular characterization of sheep ruminal enrichments that detoxify pyrrolizidine alkaloids by denaturing gradient gel electrophoresis and cloning. Microb. Ecol. 54: 264-275.

Shabat, S.K.B., Sasson, G., Doron-Faigenboim, A., Durman, T., Yaacoby, S., Miller, M.E.B., White, B.A., Shterzer, N. and Mizrahi, I. (2016) Specific microbiome-dependent mechanisms underlie the energy harvest efficiency of ruminants. ISME J.

Shiffman, M.E., Soo, R.M., Dennis, P.G., Morrison, M., Tyson, G.W., and Hugenholtz, P. (2017) Gene and genome-centric analyses of koala and wombat fecal microbiomes point to metabolic specialization for Eucalyptus digestion. PeerJ 5: e4075. 
786

787

788

789

790

791

792

793

794

795

796

797

798

799

800

801

802

803

804

805

806

807

808

809

810

811

812

813

814

815

816

817

818

819

820

821

822

823

824

825

826

827

828

Shipley, L.A., Forbey, J.S., and Moore, B.D. (2009) Revisiting the dietary niche: when is a mammalian herbivore a specialist? Integr. Comp. Biol. 49: 274-290.

Snipes, R.L., Snipes, H., and Carrick, F.N. (1993) Surface Enlargement in the Large-Intestine of the Koala (Phascolarctos cinereus)-Morphometric Parameters. Aust. J. Zool. 41: 393-397.

Sonoyama, K., Fujiwara, R., Takemura, N., Ogasawara, T., Watanabe, J., Ito, H., and Morita, T. (2009) Response of gut microbiota to fasting and hibernation in Syrian hamsters. Appl. Environ. Microbiol. 75: 6451-6456.

Soo, R.M., Skennerton, C.T., Sekiguchi, Y., Imelfort, M., Paech, S.J., Dennis, P.G., Steen, J.A., Parks, D.H., Tyson, G.W. and Hugenholtz, P. (2014) An expanded genomic representation of the phylum Cyanobacteria. Genome Biol. Evol. 6: 1031-1045.

Stearns, J.C., Lynch, M.D., Senadheera, D.B., Tenenbaum, H.C., Goldberg, M.B., Cvitkovitch, D.G., Croitoru, K., Moreno-Hagelsieb, G. and Neufeld, J.D. (2011) Bacterial biogeography of the human digestive tract. Sci. Rep. 1: 170.

Stupans, I., Jones, B., and McKinnon, R.A. (2001) Xenobiotic metabolism in Australian marsupials. Comp. Biochem. Physiol. C Toxicol Pharmacol. 128: 367-376.

Sun, Y., Cai, X., Cao, J., Wu, Z. and Pan, D. (2018). Effects of 1, 8-cineole on carbohydrate metabolism related cell structure changes of Salmonella. Front. Microbiol. 9.

Suzuki, T.A. (2017) Links between Natural Variation in the Microbiome and Host Fitness in Wild Mammals. Integr. Comp. Biol. 57: 756-769.

Tucker, D.J., Wallis, I.R., Bolton, J.M., Marsh, K.J., Rosser, A.A., Brereton, I.M., Nicolle, D. and Foley, W.J. (2010) A metabolomic approach to identifying chemical mediators of mammal-plant interactions. J. Chem. Ecol. 36: 727-735.

Turnbaugh, P.J., Hamady, M., Yatsunenko, T., Cantarel, B.L., Duncan, A., Ley, R.E., Sogin, M.L., Jones, W.J., Roe, B.A., Affourtit, J.P. and Egholm, M. (2009a) A core gut microbiome in obese and lean twins. Nature 457: 480-U487.

Van Soest, P.v., Robertson, J., and Lewis, B. (1991) Methods for dietary fiber, neutral detergent fiber, and nonstarch polysaccharides in relation to animal nutrition. J. Dairy Sci. 74: 35833597.

Wallis, I.R., Nicolle, D., and Foley, W.J. (2010) Available and not total nitrogen in leaves explains key chemical differences between the eucalypt subgenera. Forest Ecol. Manag. 260: 814-821.

Wang, Q., Garrity, G.M., Tiedje, J.M., and Cole, J.R. (2007) Naive Bayesian classifier for rapid assignment of rRNA sequences into the new bacterial taxonomy. Appl. Environ. Microbiol. 73: $5261-5267$.

Whisson, D.A., Dixon, V., Taylor, M.L., and Melzer, A. (2016) Failure to Respond to Food Resource Decline Has Catastrophic Consequences for Koalas in a High-Density Population in Southern Australia. PloS one 11: e0144348.

Wickham, H. (2009) ggplot2: elegant graphics for data analysis: Springer Science \& Business Media.

Zeng, B., Han, S., Wang, P., Wen, B., Jian, W., Guo, W., Yu, Z., Du, D., Fu, X., Kong, F. and Yang, M. (2015) The bacterial communities associated with fecal types and body weight of rex rabbits. Sci. Rep. 5: 9342. 


\section{Figure 1 (on next page)}

PCoA of $\beta$ diversity of koala faecal bacterial communities from Cape Otway.

Scatterplots from (A) weighted and (B) unweighted UniFrac matrices for the combined Cape Otway koala population with diets comprising E. viminalis and E. obliqua from 2013 and 2015. PERMANOVA analysis indicated no influence of collection year on the (A) weighted UniFrac data (Pseudo $F_{1}=0.44$, PERMANOVA $P=0.81$ ). A significant influence was detected when we analysed the (B) unweighted UniFrac data (Pseudo $F_{1}=1.76$, PERMANOVA P=0.01). When diet $x$ collection years was analysed an influence was detected on community structure $\left((\mathbf{A})\right.$ weighted UniFrac, Pseudo $F_{2}=10.07$, PERMANOVA $\left.P=0.0001\right)$ and presence / absence of microbial taxa ((B) unweighted UniFrac, Pseudo $F_{2}=2.98$, PERMANOVA P=0.0001). 

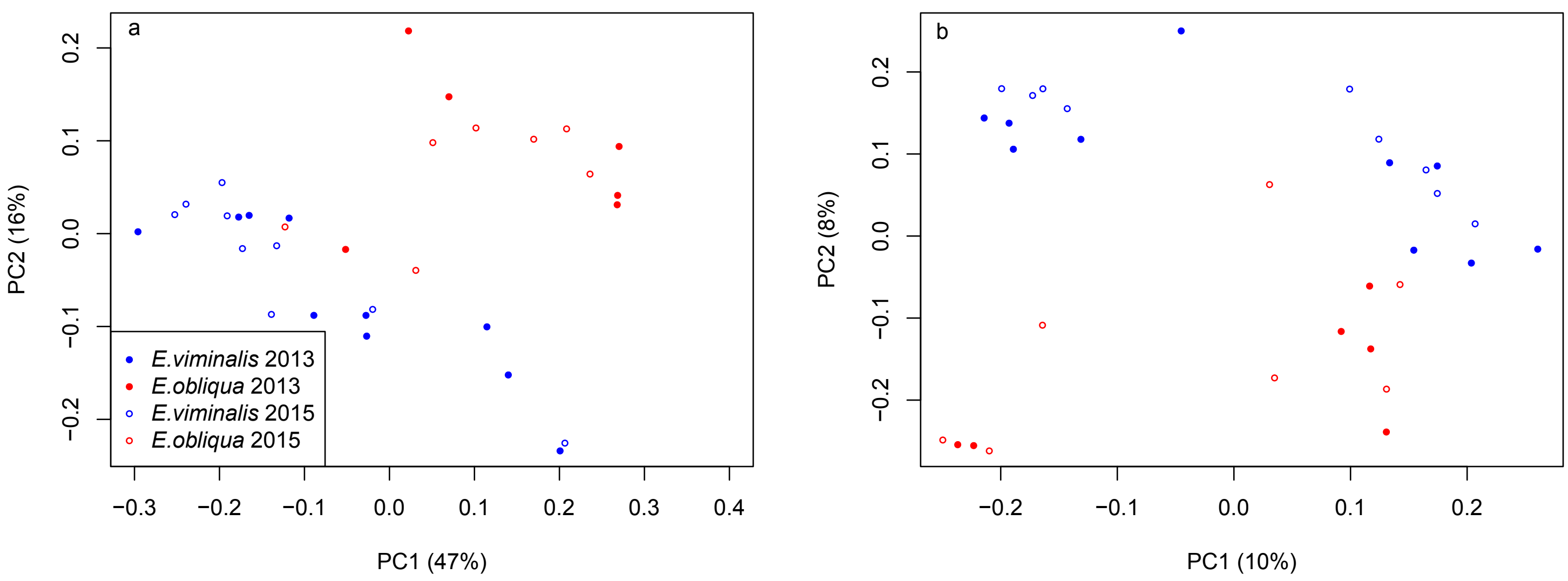
Figure 2 (on next page)

PCoA of $\beta$ diversity based on UniFrac matrices from the Cape Otway 2013 koala collection.

Scatterplots from (A) weighted and unweighted (B) UniFrac matrices for koalas with diets of E. viminalis and $E$. obliqua from the 2013 collection year. PERMANOVA assessment of the weighted and unweighted UniFrac matrices from the 2013 collection year, indicated that diet was a strong driver of both microbial community structure (relative abundance), and of microbial presence / absence during 2013 ((A) weighted UniFrac, Pseudo F1=5.88, PERMANOVA $\mathrm{P}=0.0001$; (B) unweighted UniFrac, Pseudo $\mathrm{F} 1=1.89$, PERMANOVA $\mathrm{P}=0.0001$ ). 

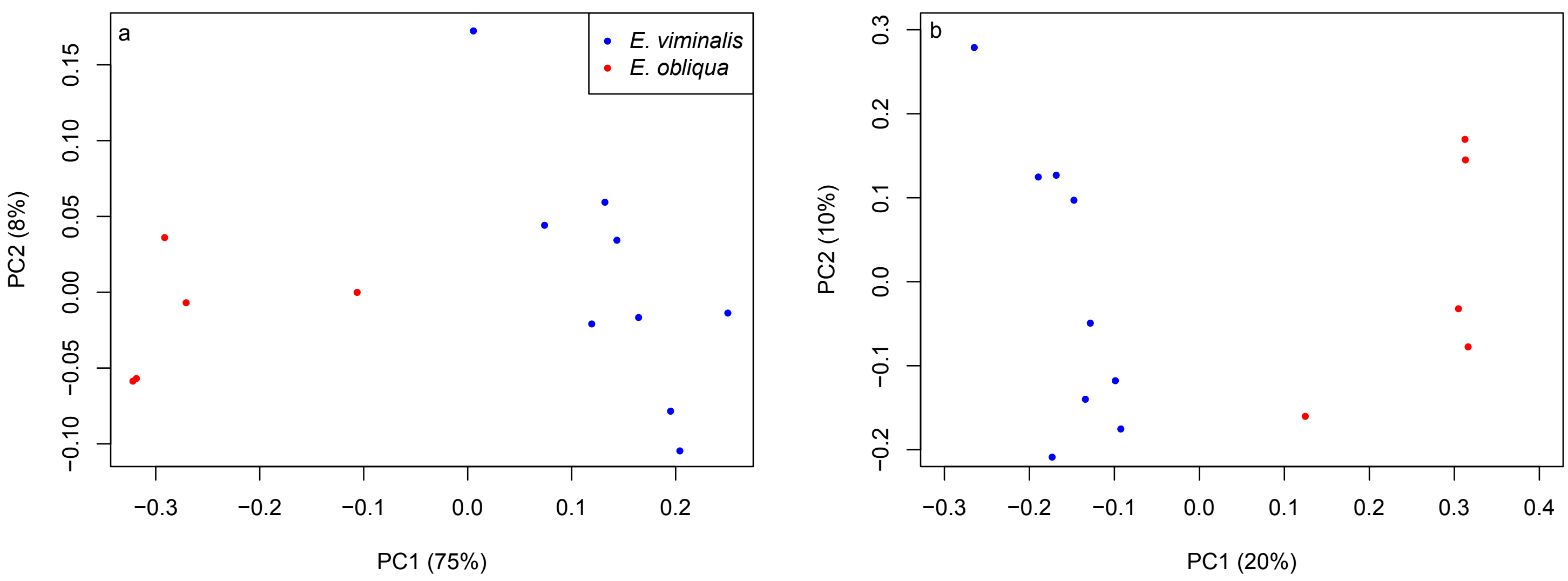
Figure 3 (on next page)

PCoA of $\beta$ diversity based on UniFrac matrices from the Cape Otway 2015 koala collection.

Scatterplots from (A) weighted and (B) unweighted UniFrac matrices for koalas with diets comprising $E$. viminalis and $E$. obliqua from the 2015 collection year. The influence of diet was significant in 2015 ((A) weighted UniFrac, Pseudo $F_{1}=8.89$, PERMANOVA P=0.0001; (B) unweighted UniFrac, Pseudo $F_{1}=2.76$, PERMANOVA P $=0.0001$ ). 
Figure 4(on next page)

Taxonomic bar charts of the most abundant genera in faecal microbiomes of koalas feeding from $E$. viminalis and E. obliqua.

Taxonomic bar charts show the dominance of Parabacteroides and Ruminococcaceae in faecal microbiomes of koalas eating E. viminalis and E. obliqua, respectively, in (A) 2013, and the dominance of Bacteroides, Parabacteroides (E. viminalis) and Ruminococcaceae (E. obliqua) in (B) 2015. 


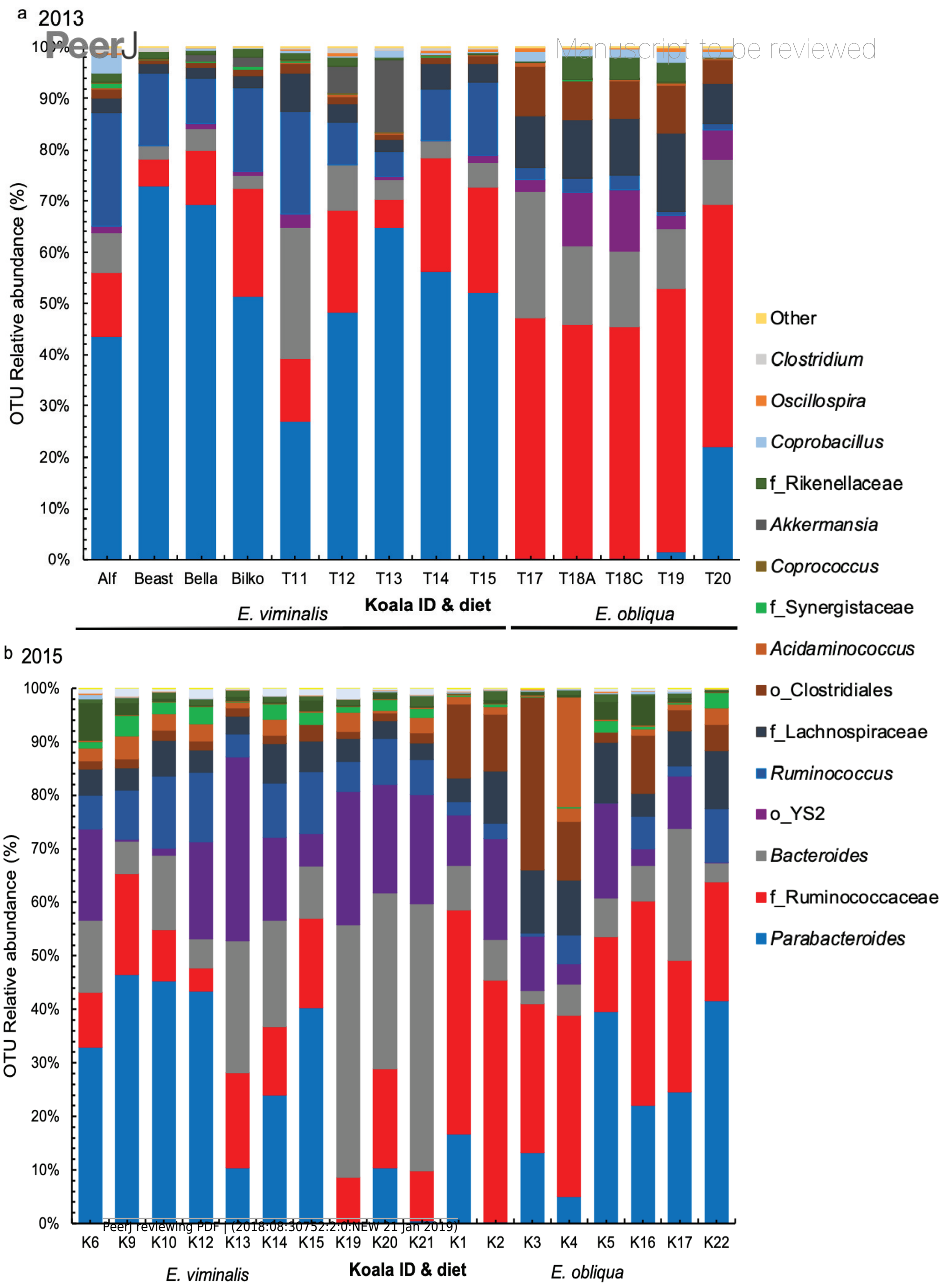


Figure $\mathbf{5}$ (on next page)

Estimated diet composition of koalas eating E. viminalis and E. obliqua.

Bar chart showing koala identity and percentage of diet species eaten for (A) $2013(n=14$ koalas) and (B) 2015 ( $n=15$ koalas) koala faecal samples. 

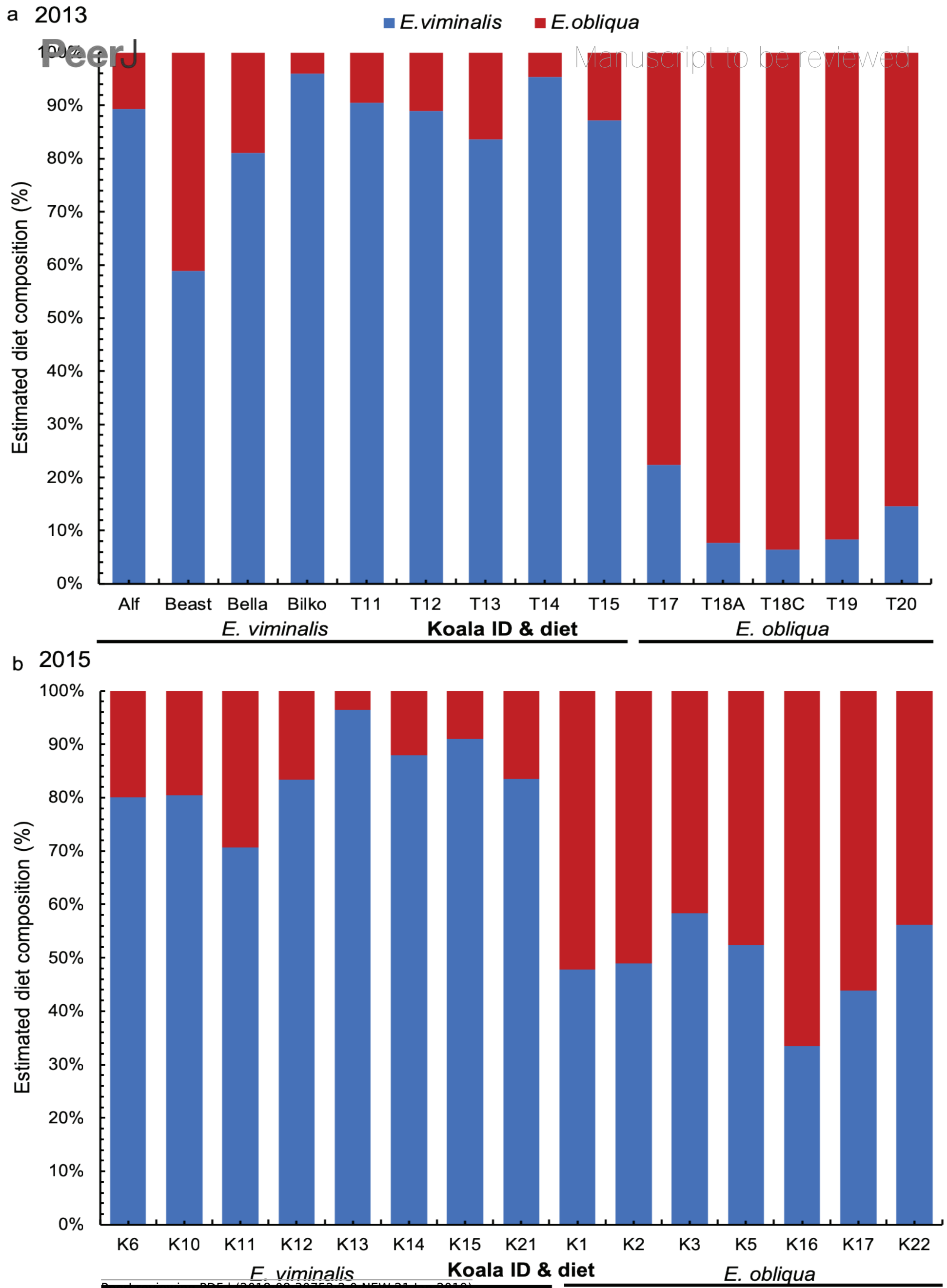


\section{Table $\mathbf{1}$ (on next page)}

Relative abundance of several bacterial phyla in faeces of koalas eating $E$. viminalis and E. obliqua in 2013 and 2015.

footnotes Values given as mean relative abundance \pm standard error (SE) across diet and collection year. Across columns, mean followed by the same letter are not significantly different at $\mathrm{P}<0.05$, using Tukey's test. 


\begin{tabular}{|c|c|c|c|c|}
\hline \multicolumn{7}{|c|}{ Frequency (\%) \pm SE } \\
\hline Taxon-phylum & E. viminalis 2013 & E. obliqua 2013 & E. viminalis 2015 & E. obliqua 2015 \\
\hline Bacteroidetes & $61 \pm 0 \mathrm{a}$ & $22 \pm 0 \mathrm{~b}$ & $48 \pm 0 \mathrm{a}$ & $27 \pm 0 \mathrm{~b}$ \\
\hline Firmicutes & $34 \pm 0 \mathrm{~b}$ & $69 \pm 0 \mathrm{a}$ & $32 \pm 0 \mathrm{~b}$ & $61 \pm 0 \mathrm{a}$ \\
\hline Cyanobacteria & $1 \pm 0 \mathrm{~b}$ & $6 \pm 0 \mathrm{ab}$ & $15 \pm 0 \mathrm{a}$ & $8 \pm 0 \mathrm{ab}$ \\
\hline Other & $1 \pm 0 \mathrm{~b}$ & $1 \pm 0 \mathrm{a}$ & $0.003 \pm 0.001 \mathrm{c}$ & $0.003 \pm 0.001 \mathrm{c}$ \\
\hline Proteobacteria & $1 \pm 0 \mathrm{a}$ & $1 \pm 0 \mathrm{a}$ & $1 \pm 0 \mathrm{a}$ & $1 \pm 0 \mathrm{a}$ \\
\hline Synergistetes & $0.003 \pm 0.001 \mathrm{~b}$ & $0.0004 \pm 0.0001 \mathrm{~b}$ & $2 \pm 0 \mathrm{a}$ & $1 \pm 0 \mathrm{~b}$ \\
\hline Verrucomicrobia & $2 \pm 0 \mathrm{a}$ & $0.00004 \pm 0.00002 \mathrm{a}$ & $1 \pm 0 \mathrm{a}$ & $1 \pm 0 \mathrm{a}$ \\
\hline
\end{tabular}




\section{Table 2 (on next page)}

Foliar nutritional quality of leaf material analysed through in vitro digestion.

footnotes Ndig (N digestibility), N (total nitrogen), AvailN (Available nitrogen), DMD (dry matter digestibility) and NDF (neutral detergent fibre). E. viminalis $(n=16)$ and E. obliqua $(n=11)$. $P$ values obtained by Student's $t$ test assessing differences between same leaf ontogeny (i.e. epicormic or adult) of both eucalypts. 
Mean foliar nutritional values $\% \pm \mathrm{SE}$

\begin{tabular}{|l|c|c|c|c|c|c|}
\hline Leaf & E. viminalis epicormic & E. obliqua epicormic & P & E. viminalis adult & E. obliqua adult & P \\
\hline Total N & $2.03 \pm 18$ & $1.3 \pm 0.4$ & 0.04 & $2 \pm 0.02$ & $1.19 \pm 3$ & 0.05 \\
\hline Ndig & $62 \pm 11$ & $6.3 \pm 2.6$ & 0.0001 & $66 \pm 3$ & $15 \pm 5$ & 0.0001 \\
\hline AvailN & $1.30 \pm 33$ & $1 \pm 0$ & 0.001 & $1.14 \pm 0.16$ & $0.17 \pm 0.06$ & 0.001 \\
\hline DMD & $54 \pm 3$ & $31 \pm 2$ & 0.0001 & $52 \pm 0$ & $35 \pm 2$ & 0.0001 \\
\hline NDF & - & - & - & $38 \pm 2$ & $48 \pm 1$ & 0.003 \\
\hline
\end{tabular}

1 